\title{
Money Market Fund Risk Measurement
}

\author{
Xue Zeng
}

School of Economics, Shanghai University, Shanghai, China

734133869@qq.com

Keywords: VaR; Money market fund; Garch

\begin{abstract}
This paper use VaR to measure the risk of money market fund. And VaR has many methods to calculate, this paper choose only one method which is more appropriate to our money market fund. First part is background and meaning, point out the importance of risk measurement. Second part is about past research of risk measurement in domestic and oversea. And then present VaR, include theory and method, and choose the appropriate method to calculate VaR of sample money market fund. After empirical analysis, found that $\mathrm{VaR}$ is appropriate to money market fund.
\end{abstract}

\section{我国货币市场基金风险度量}

\author{
曾雪 \\ 上海大学 经济学院, 中国上海 201900 \\ 734133869@qq.com
}

摘要：本文用 $V a R$ 模型对我国货币市场基金进行度量，探讨 $V a R$ 在我国货币市场中运用的可 行性。本文首先阐述了课题的研究背景及意义，指出在货币市场基金快速发展的同时，加强 对其风险管理的重要性。接着对我国货币市场基金的发展过程及风险控制现状做了介绍, 指 出我国货币市场基金目前面临的风险及风险控制的重要性。然后对 GARCH - 正态模型做了详 细的介绍,主要包括其计算原理和计算方法。然后结合我国货币市场基金的特点,采用了 GARCH 一正态模型来计算样本基金的 VaR 值，最后经过实证分析，认为将 VaR 模型运用到我国货币 市场基金的风险度量中时合适的。

关键词: VaR；货币市场基金；风险度量；GARCH

\section{1. 引言}

近年来，我国货币市场基金发展迅猛，由最开始的一支货币基金到现在的上百种，由最初的 几百亿到现在的几千亿，货币基金发展到了一个较高的程度，另外结合国外的货币市场基金 可以预见，我国的货币市场基金有着很大的发展空间。但在货币市场基金不断壮大的同时， 风险度量不可忽视，因为货币市场基金有着流动性强、风险低的特点，所以投资者总是忽略 了风险存在这一事实，然而货币市场基金虽然风险较小，但在风险发生的时候，会给投资者 造成难以挽回的重大损失，所以对货币市场基金的风险度量不可忽视。

$\mathrm{VaR}$ 作为一种先进的风险衡量工具, 在理论和实践上都得到了大量运用与论证。VaR最初是 在1989年, 由J.P. Morgan在其风险控制模型Risk Metrics中首次提出, 此后便得到了高度关注, 关于VaR的研究层出不穷, J.D. Morgan (1996) ${ }^{[1]}$ 引入t分布、广义误差分布、混合正态分布及 Johnson分布等方法改进了标准分布方法对于厚尾性的估计,出现了Delta类和Gamma类等模 型; Duffie. D. PanJ.(1997) ${ }^{[2]}$ 是关于VaR的一个十分全面而且深刻的理论综述,它不仅全面介绍 了 $\mathrm{VaR}$ 的基本概念、产生背景和各种计算方法,还对 $\mathrm{VaR}$ 在股票市场中的实际应用做了较为详 细的介绍; PenzaP,BansalV.K.(2001) ${ }^{[3]}$ 着重介绍了VaR在测度市场风险方面的应用, 并对比分 析了各种 VaR 计算模型在测度市场风险中的运用; Thomas J. Linsmeier 和 Neil D.Pearson （2000）[4]系统介绍了 $\mathrm{VaR}$ 的概念和方法, 并详细介绍了计算 $\mathrm{VaR}$ 的三种方法, 将每种 
方法运用到实际案例中, 进行对比分析, 较详细的分析了各个方法的优点与缺点, 以及各个 方法在测量市场风险中的实际运用。在2001年, 巴塞尔委员会发布新巴塞尔资本协议草案, 宣布引入 VaR 风险测量方法, 并将其运用于银行的风险管理。自此, VaR进一步得到了国内 外学者的关注, 更多的研究者对 VaR进行理论研究和实证研究, 不断地完善着这一风险模型。 随着 $\mathrm{VaR}$ 在全球金融市场上广泛的运用, 以及对其越来越多的更深入的研究, 我国的学者对 VaR也引起了重视, 对VaR 的研究也逐渐增多。郑文通 $(1997)^{[7]}$ 是我国最早对VaR进行研究的 学者, 较为详细的介绍了 $\mathrm{VaR}$ 的产生背景、基本原理、计算方法和用途等, 并提出了将 $\mathrm{VaR}$ 引入中国的必要性; 在后续的研究中, 国内学者逐渐将 $\mathrm{VaR}$ 运用到实证研究中, 例如王美今, 王华 $(2002)^{[8]}$ 通过对上海股市的实证研究得出: 正确假设收益率的分布是正确计算 $\mathrm{VaR}$ 值的前 提, 由于收益率分布普遍存在着非正态的情况,一般的GARCH模型可能会低估风险,因此必须 选择能够准确描述收益率尾部分布特点的模型;

另外还有其他学者用其它的方法来计算 $\mathrm{VaR}$, 如欧立辉 (2006) ${ }^{[9]}$ 在分析证券基金的风险特点和 统计特征时,采用了厚尾分布以及 GARCH模型来计算其样本的 VaR值, 通过比较得出了 GED 分布假设下的GARCH模型方法是所用到的分析VaR计算方法中最优的; 另有学者利用 $V a R$ 来 计算投资基金组合的风险值，例如周晓君，张玲，查奇芬(2007)[10]利用风险度量的VaR方法， 对投资股票的基金组合的风险进行实证研究，表明VaR可以作为基金市场风险评价的一种有 效手段，同时可以为建立有效的风险防范制度提供合理的实证基础。

\section{2. 货币市场基金定义及特点}

货币市场基金是以货币市场短期金融工具为投资对象的基金，是投资基金的品种之一。它主 要投资于现金、一年以内的银行定期存款、剩余期限在 397 天以内的债券、银行大额存单、 期限在一年以内的债券回购和期限在一年以内的央行票据等安全性较高的短期金融产品, 也 可以投资于中国证监会、中国人民银行认可的其它具有良好流动性的货币市场工具，但货币 市场基金投资组合的平均剩余期限不得超过 180 天。因此货币市场基金又被称为 “准储蓄产 品”。它具有风险较低、投资成本和投资门槛低、总费用水平较低、收益率较高、资金流动 性强、分红免税等特点:

一是风险较低。

货币市场基金的投资对象为货币市场短期金融工具，本身都具有低风险的特征，并且投资组 合的平均剩余期限较短, 一般为 90 至 180 天, 因此流动性较高, 不易受到市场波动的影响, 货币市场基金自然具有低风险性，极少部分发生发行方不能履约还本付息的情况。又因为证 监会规定货币基金不可以投资可转债、股票和信用等级在 $3 \mathrm{~A}$ 以下的债券，从而在投资范围 上规避了较高风险的产品, 较大程度的降低了风险。另外, 在欧美经济发达国家, 尤其在美 国，如果客户购买了货币市场基金的股份，不仅可以获得相应的投资收益，还可以对货币市 场基金中以股份形式持有的资产签发支票，所以风险更小。

二是总体费率水平较低。

货币市场基金总体费率（包括托管费、管理费、销售服务费、交易费等）水平在各类基金中 较低，只占资产净值的 $0.25 \%$ 1\%，一般在全部基金平均水平之下。并且货币市场基金并不 收取首次购买费用，从而降低了总体费用。

三是收益相对稳健且门槛较低。

货币市场基金普遍将每个基金单位的交易价格固定不变，通常保持为 1 元/基金单位，并建 立相关的资产计价和会计核算模式。货币市场基金的基准利率通常为 7 天通知存款收益率或 者半年定存利率，波动幅度极小。基金按每日计算基金收益，然后以份额形式定期将基金投 资收益结转到基金持有人的帐户上，通过份额增减数量反映基金持有人收益变动情况。另外 货币市场基金总体费率较低，同时货币市场基金的最低认购额较低，一般投资者都可以接受， 避免了只有机构投资者或者富裕投资者有能力参与到货币市场中进行交易。同时，发行货币 市场基金的大多为股权类、债券类基金的基金管理公司，这些公司一般同时发行管理多种基 
金，当整个基金市场行情或者某几种基金行情发生变化时，投资者可以及时把所持有的股票 基金或者债券基金转向货币市场基金，而且转换过程不收取转换费，因而降低了投资调整的 成本。总的来说货币市场基金投资门槛是各种投资理财产品中最低的。

四是流动性高。

所有货币市场基金均免收申购和赎回费率（除部分场内交易型货币基金），并且货币市场基 金投资组合的平均剩余期限较短，一般为 90 至 180 天，另外多只货币市场基金均开通赎回 $\mathrm{T}+0$ 实时到账，投资者还可以及时在债券型基金、股票型基金和货币市场基金中自由转换， 并且不收取手续费，因此流动性较其它基金更高。

五是交易方式为开放式。

相比较股票型、债券型基金既能用封闭式交易方式又能用开放式交易方式，货币市场基金只 能采用开放式交易方式，投资者可以在所持有基金的存续期内自由的追加或收回投资，加大 了货币市场基金的流通性，从而增强了货币市场基金的吸引力，正是由于这种开方式的交易 方式，货币市场基金的投资风险也进一步降低。

\section{GARCH-VaR模型介绍}

恩格尔 (Engle) 于1982年提出了自回归条件异方差模型, 简称ARCH模型 (Autoregressive Conditional Heteroskedastieity Model) , 并且成功地将该方法应用到英国的通货膨胀指数的波 动性研究中。但是在实际应用的过程中, 为了得到较好的拟合效果, $\mathrm{ARCH}$ 模型往往需要 大量的滞后阶数, 而如果滞后阶数过大, 无限制约束的估计往往会违背假设中的限定条件, 不能保证条件方差永远是正数, 从而降低了估计效率。为此, 1986年博勒斯莱 (BollerSlev) 通过增加自回归项, 把ARCH模型扩展成为广义的自回归条件异方差模型 (Generalized ARCH model），即GARCH模型。此后的研究中，针对市场冲击对资产收益的波动所造成的非对称 效应, 更多的学者对GARCH模型进行了拓展, 从而产生了EGARCH（the Exponential GARCH model）模型, TGARCH（the Threshold GARCH model）模型等一系列模型, 这一类模型可以 很好的对金融收益序列中存在的条件异方差性, 尖峰厚尾性, 波动集聚性, 杜杆效应等进行 刻画,同时能够较好地预测金融收益序列的波动性，所以,它们在金融时间序列分析中得到广 泛地应用。

GARCH模型的基本思想是：扰动项的条件方差不仅依赖于扰动项的滞后项，还受到条件方差 的滞后项的影响。在GARCH模型中, 需要考虑两个不同的设定: 一是条件均值, 二是条件方 差。标准的GARCH模型为

$y_{t}=x_{t} \times \gamma+u_{t}$

$\sigma_{\mathrm{t}}^{2}=\omega+\alpha \mu_{\mathrm{t}-1}^{2}+\beta \sigma_{\mathrm{t}-1}^{2}$

式中, $x_{\mathrm{t}}=\left(\mathrm{x}_{1+1}, \mathrm{x}_{2+\mathrm{t}}, \ldots, \mathrm{x}_{\mathrm{kt}}\right)$ 是解释变量向量, $\gamma=\left(\gamma_{1}, \gamma_{2}, \ldots, \gamma_{\mathrm{k}}\right)$ 是系数向量。式 $(2-2)$ 是条件方差

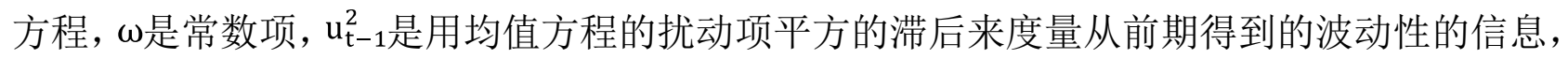

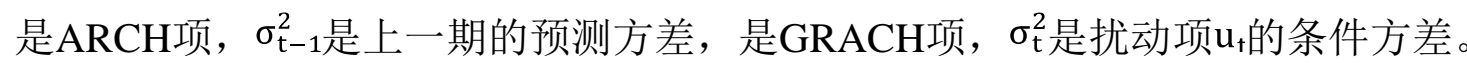

$\alpha$ 是 $\mathrm{ARCH}$ 项的系数, 反应的是滞后期的波动对市场影响的速度和长度, $\alpha$ 越大, 速度越快, 波动性越大。 $\beta$ 是 $\mathrm{GARCH}$ 项的系数, 反应的是波动持续时间的长短, $\beta$ 系数越大, 表示往期波 动对条件方差的冲击时间更长。 GARCH 类模型较其它方法相比有较大的突破, 该方法能够 对不同事件所引发的波动赋予不同的权重, 从而强化近期事件的影响, 弱化前期事件的影响, 而且该方法还可以很好的描述金融时间序列的波动集聚性特征，从而避免了同方差假定的不 合理性，更加贴近金融时间序列的真实情况。 


\section{4. 样本与数据及置信度的选取}

4.1. 样本与数据的选取

由于我国货币市场基金数量众多，很难一一进行分析，为了让分析结果更具代表性，本文选 取了成立时间较早，资产在10亿以上的货币基金。这样符合条件的货币基金有11支，但有两 家基金公司旗下的货币市场基金 $\mathrm{A}$ 和基金 $\mathrm{B}$ 同时符合条件，而货币市场基金 $\mathrm{A}$ 和基金 $\mathrm{B}$ 是同时 操作的, $\mathrm{A}$ 类基金主要是针对中小投资者发行的, $\mathrm{B}$ 类基金主要是面向机构投资者或者资产 较高的个人投资者发行的，具体由投资者在交易账户中保留的基金份额决定。在这两组基金 中，本文选取了 $\mathrm{B}$ 类基金。这样，符合条件的样本基金共有9支，具体情况如表4-1所示。本文 选取的时间跨度为2012年1月1日至2014年1月1日，选取的收益率为七日年化收益率。样本基 金的数据来源于锐思数据网（www.resset.cn）以及各个基金公司的网站。

表3-1 样本基金基本信息

\begin{tabular}{|l|l|l|l|}
\hline 基金代码 & 基金简称 & 发行日期 & 资产规模（亿元） \\
\hline 080011 & 长盛货币 & $2005-12-12$ & 10.31 \\
\hline 530002 & 建信货币 & $2006-4-25$ & 179.97 \\
\hline 660107 & 农银货币B & $2010-11-23$ & 47.69 \\
\hline 217014 & 招商现金B & $2009-12-1$ & 129.39 \\
\hline 482002 & 工银货币 & $2006-3-20$ & 664.99 \\
\hline 003003 & 华夏现金A & $2004-4-7$ & 960.47 \\
\hline 050003 & 博时现金 & $2004-1-16$ & 305.46 \\
\hline 091005 & 大成货B & $2005-6-3$ & 41.5 \\
\hline 213909 & 宝盈货币B & $2009-8-5$ & 95.41 \\
\hline
\end{tabular}

\section{2. 置信度的选取}

置信度是指根据现有的 $\mathrm{VaR}$ 值，对于预期可能发生的损失小于这个限额的把握程度，它的 选取没有特定的标准，取决于想要表达的目的。选取过低，准确性下降，不能保证监管控制 整个金融体系的稳定; 选取的过高, 又会导致资本充足率过高, 影响金融机构的投资经营效 益，降低了投资机构的盈利空间。具体实践中，各个金融机构依据各自不同的需求，选取不 同的置信度。巴塞尔委员会处于稳健性考虑，选取了 $99 \%$ 的置信度，富士银行、信孚银行、 樱花银行也选择了 $99 \%$ 的置信度, 花旗银行选择 95.4\%, 美洲银行、RiskMetrics与 J.P 摩根 则选择 $95 \%$ 。不同的置信度下计算出的 $\mathrm{VaR}$ 值完全不同, 也不具备可比性，因此不同的需求 下会选择不同的置信度。如果是激进型的投资者, 则可能会选取较低的置信度, 同时也会承 担较大的风险; 如果是稳健型的投资者, 则可能会选择较高的置信度, 降低风险发生的可能 性。本文参考了大多数金融机构的置信度后，选取了95\%的置信区间。

\section{5. 样本数据的基本分析}

\section{1 样本数据统计量的分析}

要对样本数据进行描述性的统计分析,我们就要计算样本基金数据的各项统计量,包括各个样 本收益率的均值、标准差、偏度、峰度这几个统计特征以及正态性检验的JB一统计量,其中均 值、标准差、偏度、峰度可以通过Excel软件求得, JB统计量可以通过EviewS软件求得。下面 这几种统计量进行简单的介绍:

偏度 $S$ : 随机变量 $X$ 的偏度用 $X$ 的标准化变量的三阶中心矩来表示, 用字母 $S$ 表示, 其表达公式为 $\mathrm{S}=\frac{\mathrm{E}\left(\mathrm{X}-\mathrm{u}_{\mathrm{x}}\right)^{3}}{\sigma_{\mathrm{x}}^{3}}$

偏度反映了随机变量函数曲线平均值两边的对称偏斜性, 当随机变量X服从正态分布时, 偏度 值为零, 曲线两侧尾部的长度对称。如果偏度值大于零, 此时数据位于均值右边的比位于左 边的少, 曲线表现为分布右边的尾部要比左边的尾部长; 如果偏度值小于零, 情况则相反。 
峰度 $\mathrm{K}$ :随机变量 $\mathrm{X}$ 的峰度用 $\mathrm{X}$ 的标准化变量的四阶中心矩来表示,用字母 $\mathrm{K}$ 表示, 其表达式为: $\mathrm{K}=\frac{\mathrm{E}\left(\mathrm{X}-\mathrm{u}_{\mathrm{X}}\right)^{4}}{\sigma_{\mathrm{x}}^{4}}$

峰度反映了概率密度曲线在众数附近的峰值的尖峭程度, 当随机变量X服从正态分布时, 峰 度值为 3 。如果峰度值大于 3 , 表示函数分布较陡峭, 数据比较集中, 有着比正态分布更长的 尾部; 如果峰度值小于 3 , 情况则相反。

JB 统计量: JB统计量是一种检验序列是否为正态分布的方法, 它度量的是序列的偏度值和峰 度值与相应的正态分布值的差异, 其表达式为: $J B=\frac{N-n}{6}\left[S^{2}+\frac{1}{4}(K-3)^{2}\right]$

其中, $\mathrm{N}$ 为样本容量, $\mathrm{n}$ 为用于构造样本序列的被估计系数的个数, $\mathrm{S}$ 为随机变量 $\mathrm{x}$ 的偏度, $\mathrm{K}$ 为随机变量 $\mathrm{X}$ 的峰度。当随机变量 $\mathrm{X}$ 服从正态分布时, JB统计量服从自由度为 2 的卡方分布。 从表4-2可以看出, 9支货币市场基金的收益率均值均为正，并且都在3\%以上，远比银行活期 存款利率要高。可以看出, 在收益上, 货币市场基金比银行活期存款要有优势。样本基金的 偏度都大于零, 但数值不是很大, 在 0.4212 到 2.8153 之间, 靠近正态分布的偏度值, 但全部样 本基金的收益率分布都呈右偏, 收益率位于均值右边的比位于左边的少, 收益率曲线的右边 尾部要比左边尾部长。大部分样本基金的峰度值都大于 3 , 表明样本基金收益率的分布更加集 中, 有着更长的尾部。同时, JB统计量数值都较大, 远远高于正态分布下的数值。通过以上 分析, 可以看出样本基金的收益率不显著服从正态分布, 一定程度上存在着右偏和尖峰厚尾 的倾向。同时, 从ADF检验中可以发现样本数据的稳定性不是很好, 说明样本收益序列并非 完全服从正态分布。因此, 用历史模拟法或蒙特卡罗模拟法都不能很好地描述样本基金收益 率的特征, 故采用 $\mathrm{GARCH}$ 模型来计算样本基金的VaR值。

表4-1 样本基金收益率的统计结果

\begin{tabular}{|l|l|l|l|l|l|l|}
\hline 样本基金 & 均值 $(\%)$ & 标准差 & 峰度 & 偏度 & JB & ADF \\
\hline 长盛货币 & 3.797452991 & 0.009051356 & 0.458565099 & 0.961580594 & 94.50428 & -3.45573 \\
\hline 建信货币 & 4.153247863 & 0.012268096 & 3.880063494 & 1.604710518 & 608.5992 & -5.149151 \\
\hline 农银货币B & 4.387042735 & 0.011150379 & 3.927598111 & 1.42118611 & 563.6017 & -5.343854 \\
\hline 招商现金B & 4.365487179 & 0.008093009 & -0.487835662 & 0.453726298 & 25.91554 & -1.950487 \\
\hline 工银货币 & 4.205094017 & 0.008193179 & -0.522455225 & 0.441427902 & 25.70286 & -1.876285 \\
\hline 华夏现金A & 4.289692308 & 0.007085403 & 1.366088639 & 0.889556591 & 120.8 & -3.660067 \\
\hline 博时现金 & 4.208136752 & 0.009359462 & 5.597871488 & 1.832071582 & 1073.661 & -4.244908 \\
\hline 大成货B & 3.924119658 & 0.007435846 & 3.122722345 & 1.032791136 & 335.5774 & -4.956726 \\
\hline 宝盈货币B & 4.276376068 & 0.013190115 & 12.70037386 & 2.815343194 & 4627.47 & -6.308761 \\
\hline
\end{tabular}

5.2 基于 $\mathrm{GARCH}$ 一正态模型的统计分析

通过以上对样本数据统计量的分析，可以看出我国货币市场基金并不完全服从正态分布，而 是存在一定右偏和尖峰厚尾现象, 所以采用 $\mathrm{GARCH}$ 一正态模型来对样本基金数据进行计算分 析。另外, 为了判断模型是否合适, 本文选用了 SC准则和AIC准则来进行检测, SC值和AIC 值越小, 模型越精确。SC值和AIC值通过Eviews软件计算得出。 
表4-2 GARCH一正态模型的统计结果

\begin{tabular}{|l|l|l|l|l|l|}
\hline 样本基金 & $\omega$ & $\alpha$ & $\beta$ & AIC & SC \\
\hline 长盛货币 & 0.000525 & 0.006254 & 0.005683 & 0.934353 & 0.964284 \\
\hline 建信货币 & 0.000245 & 0.008835 & 0.004462 & 0.680159 & 0.71009 \\
\hline 农银货币B & 0.000897 & 0.013616 & 0.012881 & 0.825187 & 0.855118 \\
\hline 招商现金B & $8.34 \mathrm{E}-05$ & 0.039908 & 0.017418 & -1.454288 & -1.424357 \\
\hline 工银货币 & $5.69 \mathrm{E}-05$ & 0.030129 & 0.01305 & -1.042011 & -1.01208 \\
\hline 华夏现金A & 0.000281 & 0.005604 & 0.009186 & -0.380853 & -0.350922 \\
\hline 博时现金 & 0.00011 & 0.003388 & 0.001565 & 0.295048 & 0.324979 \\
\hline 大成货B & 0.000728 & 0.034544 & 0.023799 & 0.403126 & 0.433057 \\
\hline 宝盈货币B & 0.000233 & 0.006671 & 0.002822 & 0.926933 & 0.956864 \\
\hline
\end{tabular}

从以上统计量可以看出, 样本基金的ARCH项和 GARCH项大部分在 $95 \%$ 的置信水平下显著, 并且 $\mathrm{SC}$ 值和 $\mathrm{AIC}$ 值也都较小，这表明采用 $\mathrm{GARCH}$ 一正态模型对样本基金进行计算分析是合适 的。

\section{VaR值计算与小结}

在确定模型的选取合适后，本文运用 $\mathrm{GARCH}$ 一正态模型对样本基金进行 VaR值的计算，置信 水平为 $95 \%$ 。从表 5-1可以看出, 在 $95 \%$ 的置信水平下, 基于 $\mathrm{GARCH}$ 一正态模型计算出的样本 基金的 $\mathrm{VaR}$ 值都很小，从效果来看， $\mathrm{GARCH}$ 一正态模型计算样本基金的 $\mathrm{VaR}$ 值是合适的。

表5-1 样本基金的日VaR值

\begin{tabular}{|l|l|}
\hline 样本基金 & $\mathrm{VaR}$ \\
\hline 长盛货币 & 0.000354116 \\
\hline 建信货币 & 0.000172605 \\
\hline 农银货币B & 0.000601887 \\
\hline 招商现金B & $1.9131 \mathrm{E}-05$ \\
\hline 工银货币 & 0.004551861 \\
\hline 华夏现金A & 0.164879748 \\
\hline 博时现金 & $5.71992 \mathrm{E}-05$ \\
\hline 大成货B & 0.000400705 \\
\hline 宝盈货币B & 0.000192709 \\
\hline
\end{tabular}

本文通过对选取的9支样本基金进行实证分析，通过偏度、峰度等统计量的分析及基于 GARCH 一正态模型的 $\mathrm{VaR}$ 值的计算，发现我国货币市场基金的收益率序列存在一定程度上的尖峰厚 尾的特征：样本基金的偏度都大于零，虽然靠近正态分布的偏度值，但全部样本基金的收益 率分布都呈右偏, 收益率曲线的右边尾部要比左边尾部长; 大部分样本基金的峰度值都大于 3 , 表明样本基金收益率的分布更加集中，有着更长的尾部。这表明我国货币市场基金的收益率 序列不完全服从正态分布。同时，通过检验分析，用GARCH一正态模型来计算时，SC和AIC 值都较小，表明模型的选取是合适的，用 $\mathrm{GARCH}$ 一正态模型来计算我国货币市场基金的VaR 值是可行的。 


\section{参考文献}

[1] J.P. Morgan. RiskMetries Technical Document. NewYork: J. P. Morgan (Fourth Edition), www.jpmorgan.com/RiskManagement/RiskMetrics/RiskMetrics.1996: 1-296

[2] DuffieD, PanJ.An overview of value at Risk. Journal of Derivatives, 1997,4(3):7-49

[3] Penza, PBansalVK. Measuring Market Risk with value at Risk. Newyork: JolmWileyandSons,Inc, 2001

[4] Thomas J. Linsmeier, Neil D. Pearson. Value at Risk. Financial Analysts Journal, 2000, Vo1.56,No.2: P47-67

[5] Fabio C. Bagliano, Carlo A. Favero. Measuring monetary policy with VAR models: An evaluation. European Economic Review,1998,42:P1069-1112

[6] Jorion. P risk: Measuring the risk in value at risk. Financial Analysts Journal,1996

[7] 郑文通. 金融风险管理的VAR方法及其应用 $[J]$ ，国际金融研究，1997，(09）

[8] 王美今，王华. 基于 $\mathrm{GARCH}$ 一 $\mathrm{t}$ 的上海股票市场价值分析 $[\mathrm{J}]$, 数量经济技术经济 究, 2002, (3)

[9] 欧立辉. 基于 $\mathrm{CARCH}$ 模型的 VaR 度量证券投资基金风险实证研究 $[\mathrm{J}]$, 湖南农业大学学 报, 2006,(6)

[10］周晓君, 张玲, 查奇芬. 基金投资组合风险评价中 $V a R$ 的应用研究 $[\mathrm{J}]$, 商业研究 2007, (2)

[11] 龚金萍. 基于 $\mathrm{VaR}$ 的我国货币市场基金的风险度量及绩效评价 [D] : 硕士学位论文. 江西：华东交通大学产业经济学, 2012.

[12］林沐尘，申远. 基于EGARCH-Copula模型的VaR方法在投资组合风险分析中的应用 $[\mathrm{J}]$, 金融经济, 2014，(20).

[13］陈静. 我国货币市场基金流动性风险问题研究 $[J]$, 上海金融, 2013，(10).

[14] 严伟祥, 张杰. 基于 GARCH-VaR模型的对冲基金市场风险度量研究 $[\mathrm{J}]$, 经济与管理评 论, 2013, (05).

\section{References}

[1] J.P.Morgan. RiskMetries Technical Document. NewYork: J.P. Morgan (Fourth Edition), www.jpmorgan.com/RiskManagement/RiskMetrics/RiskMetrics.1996: 1-296

[2] DuffieD, PanJ. An overview of value at Risk. Journal of Derivatives,1997,4(3): 7-49

[3] Penza, PBansalVK. Measuring Market Risk with value at Risk. Newyork: JolmWileyandSons, Inc, 2001

[4] Thomas J.Linsmeier, Neil D.Pearson. Value at Risk.Financial Analysts Journal, 2000, Vo1.56,No.2: P47-67

[5] Fabio C. Bagliano, Carlo A. Favero. Measuring monetary policy with VAR models: An evaluation. European Economic Review,1998,42:P1069-1112

[6] Jorion. P risk: Measuring the risk in value at risk. Financial Analysts Journal,1996

[7] Wentong Zheng. Financial Risk Management VAR method and its application. Studies of International Finance, 1997,(09)

[8] Meijin Wang, Hua Wang. Based on GARCH - t of Shanghai stock market value analysis .The Journal of Quantitative \& Technical Economics,2002,(3) 
[9] Lihui Ou. An Empirical Study of Securities Investment Funds Risk Measurement Model Based on VaR GARCH. Journal of Hunan Agricultural University(Social Sciences),2006,(6)

[10]Xiaojun Zhou, Ling Zhang, Qifen Cha. Application of the VaR of the Fund Portfolio Risk Assessment .Commercial Research,2007,(2)

[11]Jinping Gong. Based on $\mathrm{VaR}$ of risk measure of money market funds and performance evaluation .Master Thesis. Jiangxi: East China Jiaotong University Industrial Economics, 2012.

[12] Muchen Lin, Yuan Shen. Based on VaR Method GARCH-Copula Model Application Portfolio Risk Analysis .Finance Economy, 2014, (20).

[13]ing Chen. Study on Liquidity Risk of China's Money Market Fund. Shanghai Finance, 2013, (10).

[14]Weixiang Yan, Jie Zhang. Measurement based hedge fund market risk GARCH-VaR model. Review of Economy and Management, 2013, (05).

作者简介: 曾雪 $(1991-)$, 女, 重庆, 研究生, 产业经济学, 734133869@qq. com。 